\title{
Data-driven algorithm for the diagnosis of behavioral variant frontotemporal dementia
}

Ana L. Manera ${ }^{1}$; Mahsa Dadar ${ }^{1}$; John van Swieten ${ }^{3}$, Barbara Borroni ${ }^{4}$, Raquel Sanchez-Valle ${ }^{5}$, Fermin Moreno ${ }^{6}$, Robert LaForce $\mathrm{Jr}^{7}$, Caroline $\mathrm{Graff}^{8}$, Matthis Synofzik ${ }^{9}$, Daniela

Galimberti $^{10,11}$, James Rowe ${ }^{12}$, Mario Masellis ${ }^{13}$, Maria Carmela Tartaglia ${ }^{14}$, Elizabeth Finger ${ }^{15}$, Rik Vandenberghe ${ }^{16}$, Alexandre de Mendonça ${ }^{17}$, Fabrizio Tagliavini ${ }^{18}$, Isabel Santana ${ }^{19}$, Chris

Butler $^{20}$, Alex Gerhard ${ }^{21}$, Adrian Danek ${ }^{22}$, Johannes Levin ${ }^{22}$, Markus Otto ${ }^{23}$, Giovanni

Frisoni $^{24,25}$, Roberta Ghidoni ${ }^{26}$, Sandro Sorbi ${ }^{27,28}$, Jonathan D Rohrer ${ }^{29}$; Simon Ducharme ${ }^{1,2^{*}}$ and

$$
\text { D. Louis Collins }{ }^{1 *}
$$

Frontotemporal Lobar Degeneration Neuroimaging Initiative (FTLDNI) ${ }^{\dagger}$

GENetic Frontotemporal dementia Initiative (GENFI) $\$$

*These authors contributed equally to this work (shared senior authorship).

\section{Author's Affiliations:}

${ }^{1}$ McConnell Brain Imaging Centre, Montreal Neurological Institute, McGill University, Montreal, Quebec (QC), Canada. 3801, University, Montreal, Quebec, H3A 2B4.

${ }^{2}$ Department of Psychiatry, McGill University Health Centre. 1025 Pine Avenue, Montreal, Quebec, Canada, H4A 1ª1.

${ }^{3}$ Department of Neurology, Erasmus Medical Center, Rotterdam, The Netherlands

${ }^{4}$ Centre for Neurodegenerative Disorders, Department of Clinical and Experimental Sciences, University of Brescia, Brescia, Italy

5 Alzheimer's disease and Other Cognitive Disorders Unit, Neurology Service, Hospital Clínic, Institut d’Investigacións Biomèdiques August Pi I Sunyer, University of Barcelona, Barcelona, Spain

${ }^{6}$ Cognitive Disorders Unit, Department of Neurology, Donostia University Hospital, San Sebastian, Gipuzkoa, Spain

${ }^{7}$ Clinique Interdisciplinaire de Mémoire, Département des Sciences Neurologiques, CHU de Québec, and Faculté de Médecine, Université Laval, Quebec, Canada

8 Department of Geriatric Medicine, Karolinska University Hospital-Huddinge, Stockholm, Sweden 
${ }^{9}$ Department of Neurodegenerative Diseases, Hertie-Institute for Clinical Brain Research and Center of Neurology, University of Tübingen, Tübingen, Germany

${ }^{10}$ Fondazione IRCCS Ca’ Granda Ospedale Maggiore Policlinico, Neurodegenerative Diseases Unit, Milan, Italy

${ }^{11}$ LANE - Laboratory of Alzheimer's Neuroimaging and Epidemiology, IRCCS Istituto Centro San Giovanni di Dio Fatebenefratelli, Brescia, Italy

${ }^{12}$ Department of Clinical Neurosciences, University of Cambridge, Cambridge, UK

${ }^{13}$ Sunnybrook Health Sciences Centre, Sunnybrook Research Institute, University of Toronto, Toronto, Canada

${ }^{14}$ Toronto Western Hospital, Tanz Centre for Research in Neurodegenerative Disease, Toronto, Ontario, Canada

${ }^{15}$ Department of Clinical Neurological Sciences, University of Western Ontario, London, ON, Canada

${ }^{16}$ Laboratory for Cognitive Neurology, Department of Neurosciences, KU Leuven, Leuven, Belgium

${ }^{17}$ Faculty of Medicine, University of Lisbon, Lisbon, Portugal

${ }^{18}$ Fondazione Istituto di Ricovero e Cura a Carattere Scientifico Istituto Neurologico Carlo Besta, Milan, Italy

${ }^{19}$ Neurology Department, Centro Hospitalar e Universitário de Coimbra, Coimbra, Portugal

${ }^{20}$ Department of Clinical Neurology, University of Oxford, Oxford, UK

21 Institute of Brain, Behaviour and Mental Health, The University of Manchester, Withington, Manchester, UK

22 Neurologische Klinik und Poliklinik, Ludwig-Maximilians-Universität, Munich, German Center for Neurodegenerative Diseases (DZNE), Munich, Germany

${ }^{23}$ Department of Neurology, University Hospital Ulm, Ulm, Germany

${ }^{24}$ LANE - Laboratory of Alzheimer's Neuroimaging and Epidemiology, IRCCS Istituto Centro San Giovanni di Dio Fatebenefratelli, Brescia, Italy

${ }^{25}$ Memory Clinic and LANVIE-Laboratory of Neuroimaging of Aging, University Hospitals and University of Geneva, Geneva, Switzerland

${ }^{26}$ Molecular Markers Laboratory, IRCCS Istituto Centro San Giovanni di Dio Fatebenefratelli, Brescia, Italy 
27 Department of Neuroscience, Psychology, Drug Research and Child Health, University of Florence, Florence, Italy

${ }^{28}$ Department of Neuroscience, Psychology, Drug Research, and Child Health, University of Florence, Florence, Italy

${ }^{29}$ Department of Neurodegenerative Disease, Dementia Research Centre, UCL Institute of Neurology, Queen Square, London, UK

$\dagger$ Data used in preparation of this article were obtained from the Frontotemporal Lobar Degeneration Neuroimaging Initiative (FTLDNI) database (http://4rtni-ftldni.ini.usc.edu/ ). The investigators at NIFD/FTLDNI contributed to the design and implementation of FTLDNI and/or provided data but did not participate in analysis or writing of this report (unless otherwise listed).

${ }^{\ddagger}$ List of other GENFI Consortium Members in Appendix 


\section{Abbreviations}

FTD: frontotemporal dementia

GRN: progranulin

MAPT: microtubule-associated protein tau

C9orf72 : chromosome 9 open reading frame 72

bvFTD: behavioural variant of frontotemporal dementia

MRI: magnetic resonance imaging

CNCs: cognitively normal controls

DBM: deformation-based morphometry

FTLDNI: frontotemporal lobar degeneration neuroimaging initiative

T1-w: T1 weighted

GENFI: Genetic frontotemporal dementia initiative

MMSE: Mini mental state examination

MOCA: Montreal cognitive assessment

FTLD-CDR: Frontotemporal lobar degeneration Clinical Dementia Rating score

CGI: Clinical global impression

FRS: Frontotemporal dementia rating scale

FDR: False Discovery Rate

PCA: Principal component analysis

PCs: Principal components

SF: Semantic fluency

ROC: Receiver operating characteristic curves

AUC: Area under the curve 
bioRxiv preprint doi: https://doi.org/10.1101/2019.12.19.883462; this version posted December 20, 2019. The copyright holder for this preprint (which was not certified by peer review) is the author/funder, who has granted bioRxiv a license to display the preprint in perpetuity. It is made available under aCC-BY-ND 4.0 International license.

\section{LR+: positive likelihood ratio}

\section{LR-: negative likelihood ratio}




\begin{abstract}
INTRODUCTION: Brain structural imaging is paramount for the diagnosis of behavioral variant of frontotemporal dementia (bvFTD), but it has low sensitivity leading to erroneous or late diagnosis.
\end{abstract}

METHODS: A total of 515 subjects from two different bvFTD databases (training and validation cohorts) were included to perform voxel-wise deformation-based morphometry analysis to identify regions with significant differences between bvFTD and controls. A random forest classifier was used to individually predict bvFTD from morphometric differences in isolation and together with bedside cognitive scores.

RESULTS: Average ten-fold cross-validation accuracy was 89\% (82\% sensitivity, $93 \%$ specificity) using only MRI and 94\% (89\% sensitivity, 98\% specificity) with the addition of semantic fluency. In a separate validation cohort of genetically confirmed bvFTD, accuracy was 88\% (81\% sensitivity, 92\% specificity) with MRI and 91\% (79\% sensitivity, 96\% specificity) with added cognitive scores.

DISCUSSION: The random forest classifier developed can accurately predict bvFTD at the individual subject level.

\title{
Keywords
}

Frontotemporal dementia -Magnetic resonance -Deformation-based morphometry Classification- Machine learning 


\section{Introduction}

The heterogeneity of frontotemporal dementia (FTD) is frequently considered a hallmark of the disease with significant variations in heritability, pathology and clinical presentations (1). First, although most cases of FTD are sporadic, there is a positive familiar history in $30-50 \%$ of the cases, and 10-30\% are caused by an autosomal dominant mutation (most commonly progranulin -GRN-, microtubule-associated protein Tau -MAPT- and chromosome 9 open reading frame 72 C9orf72-) (2, 3). Second, in terms of the underlying pathology, there are three main groups according to the major protein involved, all of which are characterized by selective degeneration of the frontal and temporal lobes: Tau, transactive response DNA-binding protein of $43 \mathrm{kDa}$ TDP-43-, and the tumor associated protein fused in sarcoma -FUS-.(4, 5)

Finally, regarding its clinical presentation, FTD is divided into three major clinical syndromes: the behavioral variant (bvFTD) and two language variants (Semantic Dementia and Non-Fluent Primary Progressive Aphasia). As the initially focal brain involvement spreads to involve larger regions in the frontal and temporal lobes, the symptoms of the three variants described can converge (5). Yet, there is also a considerable overlap between FTD and other neurodegenerative diseases; i.e. some patients with FTD may develop motor neuron disease, or symptoms of a Parkinsonian disorder (6).

Due to the aforementioned heterogeneity in pathology and heritability, as well as the syndromic overlap with psychiatric disorders and other dementias, a confirmed bvFTD diagnosis is often difficult to achieve in the absence of a dominant genetic mutation. Indeed, although brain imaging with magnetic resonance imaging (MRI) is paramount to increase the level of diagnostic 
confidence, it lacks sensitivity, particularly in the initial stages of the disease, leading to erroneous or late diagnosis $(7,8)$.

It has been recognized that the pattern of atrophy in sporadic bvFTD differed but also shared some similarities from that observed in mutation carriers. While frontal -particularly the anterior cingulate gyrus- and anterior temporal atrophy was associated to sporadic bvFTD, genetic variants showed distinct but overlapping patterns of atrophy (9-11). In MAPT mutation carriers, atrophy predominantly affects the anterior and medial temporal lobes, orbitofrontal lobe and insula; in $G R N$ mutation carriers atrophy in the dorsolateral and ventromedial prefrontal, superolateral temporal and lateral parietal lobes as well as the anterior cingulate, insula, precuneus and striatum has been described; and C9orf72 carriers showed relatively widespread cortical atrophy including posterior areas, and with particular involvement of the thalamus and superoposterior cerebellum (11).

Lately, machine learning techniques have been applied to distinguish between bvFTD and Cognitively Normal Subjects (CNCs), Alzheimer Disease or other psychiatric and neurologic disorders on an individual level using MRI based features (12-20). Studies vary greatly on the subjects included and the methodology. In the present study, we developed a Random Forest classifier (21) using features derived from Deformation Based Morphometry (DBM) maps to identify bvFTD subjects from CNCs. To ensure the generalizability of the results, the machine learning model was trained on a mainly sporadic cohort and tested in a held-out population of genetic bvFTD, therefore relying on one of the gold standards for bvFTD diagnosis (i.e., definite bvFTD)(7). 


\section{Materials and Methods}

\subsection{Participants}

A total of 515 subjects were examined in this study. The first cohort ('training cohort') included bvFTD patients and CNCs from the Frontotemporal Lobar Degeneration Neuroimaging Initiative (FTLDNI) database who had T1-weighted (T1w) MRI scans matching with each clinical visit. Data was accessed and downloaded through the LONI platform in August 2018. The inclusion criteria for bvFTD patients was a diagnosis of possible or probable bvFTD according to the FTD consortium criteria (7), resulting in 70 patients with bvFTD and 123 CNCs in our study. The FTLDNI was funded through the National Institute of Aging and started in 2010. The primary goals of FTLDNI are to identify neuroimaging modalities and methods of analysis for tracking frontotemporal lobar degeneration (FTLD) and to assess the value of imaging versus other biomarkers in diagnostic roles. The project is the result of collaborative efforts at three sites in North America. For up-to-date information on participation and protocol, please visit: http://4rtni-ftldni.ini.usc.edu/

The second cohort ('validation cohort') included bvFTD patients and CNCs from the third data freeze (12/2017) of the Genetic Frontotemporal Dementia Initiative 2 (GENFI2http://genfi.org.uk/) (22), which consists of 23 centres in the UK, Italy, The Netherlands, Sweden, Portugal and Canada. GENFI2 participants included known symptomatic carriers of a pathogenic mutation in $C 9 \operatorname{orf72}, G R N$ or $M A P T$ and their first-degree relatives who are at risk of carrying a mutation, but who did not show any symptoms (i.e., presymptomatic). Non-carriers were first-degree relatives of symptomatic carriers who did not carry the mutation. The inclusion and exclusion criteria are described in detail elsewhere (22). Since the aim of the present study 
was to differentiate bvFTD patients from CNCs, presymptomatic carriers and symptomatic carriers whose clinical diagnosis was other than bvFTD were excluded. Non-carriers were considered as CNCs for the purpose of this study. This validation cohort contained 75 patients with bvFTD and 247 CNCs.

\subsection{Clinical assessment}

All FTLDNI subjects were regularly assessed every six-months for clinical characteristics (motor, non-motor and neuropsychological performance) by site investigators. Neuropsychological assessment included Mini Mental State Examination (MMSE), Montreal Cognitive Assessment (MOCA), Frontotemporal lobar degeneration clinical dementia rating (FTLD-CDR), Clinical Global Impression (CGI), verbal fluency, Frontotemporal dementia rating scale (FRS) amongst other cognitive and functional scores.

\subsection{Image acquisition and preprocessing}

For the FTLDNI training cohort, 3.0T MRIs were acquired at three sites. In all sites, a volumetric MPRAGE sequence was used to acquire T1w images of the entire brain. The acquisition parameters of the T1w images, using volumetric MPRAGE sequence, were RT/ET/IT: 2.3/3/900 ms, flip angle $9^{\circ}$, matrix 256x240, slice thickness $1 \mathrm{~mm}$, voxel size 1x1mm.

For the GENFI2 validation cohort, participants underwent volumetric T1w MRI at multiple centers, according to the GENFI imaging protocol. Sites used different types of scanners: Siemens Trio 3T, SiemensSkyra3T, Siemens1.5T, Phillips3T, General Electric (GE) 1.5T and 
GE 3T. Scan protocols were designed at the outset of the study to ensure adequate matching between the scanners and image quality control.

The T1w scans of the subjects were pre-processed through our longitudinal pipeline (23) that included image denoising (24), intensity non-uniformity correction (25), and image intensity normalization into range (0-100) using histogram matching. Each native T1w volume from each timepoint was linearly registered first to the subject-specific template which was then registered to the ICBM152 template $(26,27)$. All images were then non-linearly registered to the ICBM152 template using ANTs diffeomorphic registration pipeline (28). The images were visually assessed by two experienced raters to exclude cases with significant imaging artifacts (e.g. motion, incomplete field of view) or inaccurate linear/nonlinear registrations. This visual assessment was performed without any knowledge of the diagnosis. Out of 1724 scans, only 43 $(2.5 \%, 36$ scans in GENFI2, and 7 in FTLDNI) did not pass this visual quality control. For the purpose of this study, scans from subjects other than bvFTD or CNCs, or those that did not have a matching clinical visit were excluded from this analysis. This resulted in a total of 515 subjects that were included in this study.

\subsection{Deformation based morphometry}

DBM analysis was performed using Montreal Neurological Institute (MNI) MINC tools (23). The principle of DBM is to warp each individual scan to the template by introducing highdimensional deformations $(29,30)$. Then, the morphological differences between the two are encoded in the deformations estimated for the warp. The local deformation obtained from the non-linear transformations was used as a measure of tissue expansion or atrophy by computing the determinant of the Jacobian at each voxel. Local contractions can be interpreted as shrinkage 
(e.g., tissue atrophy) and local expansions are often related to ventricular or sulci enlargement.

DBM was used to assess both voxel-wise and atlas-based cross-sectional group related volumetric differences.

\subsection{Classification bvFTD versus CNCs}

To obtain a region of interest reflecting the difference between bvFTD and CNCs, a voxel-wise mixed effects model analysis was performed in the training dataset to assess the pattern of volumetric change according to diagnosis. The mixed effects model included age as a continuous fixed variable and diagnosis and sex as fixed categorical variables. Subject was included as a categorical random variable. The resulting maps were corrected for multiple comparisons using False Discovery Rate (FDR) with a $P$ value $<.05$ threshold to identify regions associated with differences between bvFTD and CNCs; i.e. the diagnosis variable in the mixed effects model. A principal component analysis (PCA) was then performed on the DBM voxels within this region of interest. To avoid any leakage, only the training data was used for this PCA. Two sets of features were then used to train a random forests classifier (21) with 500 trees: 1) the first five principal components (PCs) as well as age and sex, and 2) the first five PCs, age, sex, and a neuropsychological score. The Semantic Fluency score (SF) was used as the cognitive score feature since it is a reliable simple bedside test associated with executive and language deficits in bvFTD (31) and was available for most of the subjects in both training and validation datasets. Executive deficits are considered a core characteristic of FTD, though in themselves, are insufficient to establish a diagnosis $(32,33)$. Classifications were run using DBM in isolation and $\mathrm{DBM}+\mathrm{SF}$. Ten-fold cross validation was used to assess the performance of the classifier within the training data. 
To perform classification on the held-out GENFI2 validation dataset, the coefficients calculated based on the PCA on the training dataset were used to calculate the first five PCs features for the subjects from the validation dataset. Using the random forest classifier that was trained on FTLDNI, we then classified all the subjects from the validation dataset as either bvFTD or CNCs. A probability score was also obtained from the random forest classifier, indicating the likelihood of each observation belonging to the bvFTD class. The mixed effects modelling, PCA, and random forest classification were carried out using MATLAB (version R2017b).

\subsection{Statistical analyses}

All statistical analyses were also conducted using MATLAB (version R2017b). Two-sample tTests were conducted to examine demographic and clinical variables at baseline. Categorical variables were analysed using chi-square analyses. Results are expressed as mean \pm standard deviation and median [interquartile range] as appropriate. Receiver Operator Characteristics (ROC) analysis was used to define sensitivity and specificity at different cut-points within the validation cohort. The optimal cut-point was estimated by the use of Youden index ( $\mathrm{J}=$ Sensitivity+Specificity-1). Positive and negative likelihood ratios were also estimated for different cut points.

\section{Results}

\subsection{Demographics}

Table 1 shows the demographic and cognitive testing performances in bvFTD and CNCs. There was no difference in age between bvFTD patients and CNCs (62 \pm 6 and $63 \pm 6$ years respectively, 
$P$ value $=0.36)$, but there was a higher proportion of males in bvFTD patients than CNCs $(67 \%$ vs $43 \%, P$ value $=.001)$. As expected, bvFTD subjects showed greater cognitive and functional impairment: significant differences were found between the two cohorts in MMSE, FTLD-CDR, MOCA, letter fluency Z-score and semantic fluency Z-score (all $P$ value $<.001$ ).

Demographic differences and cognitive testing performances between patients and controls for the GENFI cohort are also shown in Table 1. Considering the CNCs from this dataset comes from non-carrier members of families at risk of genetic mutation related to FTD, they were, as expected, significantly younger than bvFTD subjects. The mean age was $48 \pm 14$ years for CNCs and $64 \pm 8$ years for bvFTD $(P$ value $<.001)$. The mean estimated disease duration for the bvFTD group was 5.2 \pm 5.7 years. Compared to non-carriers, bvFTD subjects showed greater cognitive and functional impairment. Significant differences were found between the two cohorts in MMSE, FTLD-CDR, MOCA, FRS, letter fluency Z-score and semantic fluency Z-score $(P$ value $<.001)$. Regarding the mutated gene, half of the bvFTD subjects carried a C9orf72 mutation, while $22.7 \%$ and $25.3 \%$ belonged to the $M A P T$ and $G R N$ groups respectively. 
Table 1. Demographic and clinical characteristics in bvFTD and healthy controls

\begin{tabular}{|c|c|c|c|c|c|c|}
\hline & \multicolumn{3}{|c|}{$\begin{array}{c}\text { Training cohort (FTLDNI) } \\
N=193\end{array}$} & \multicolumn{3}{|c|}{$\begin{array}{c}\text { Validation cohort (GENFI) } \\
\qquad N=322\end{array}$} \\
\hline & $\begin{array}{c}\mathrm{CNCs} \\
\mathrm{N}=123\end{array}$ & $\begin{array}{c}\text { bvFTD } \\
\mathrm{N}=70\end{array}$ & P value & $\begin{array}{l}\mathrm{CNCs} \\
\mathrm{N}=247\end{array}$ & $\begin{array}{l}\text { bvFTD } \\
\mathrm{N}=75\end{array}$ & p-Value \\
\hline Age, $y$ & $63 \pm 6$ & $62 \pm 6$ & .36 & $48 \pm 14$ & $64 \pm 8$ & $<.001$ \\
\hline Male sex & $53(43 \%)$ & $47(67 \%)$ & .001 & $106(43 \%)$ & $41(55 \%)$ & .07 \\
\hline Education, y & $17.5 \pm 1.9$ & $15.6 \pm 3.4$ & $<.001$ & $13.9 \pm 3.5$ & $11.8 \pm 4.03$ & $<.001$ \\
\hline Years of onset, $y$ & - & N/A & - & - & $5.2 \pm 5.7$ & - \\
\hline MMSE score & $29.4 \pm 0.8$ & $23.6 \pm 4.9$ & $<.001$ & $29.4 \pm 1.1$ & $21.9 \pm 7.2$ & $<.001$ \\
\hline FTLD-CDR Score & $0.04 \pm 0.2$ & $6.3 \pm 3.3$ & $<.001$ & $0.21 \pm 0.7$ & $9.7 \pm 1.4$ & $<.001$ \\
\hline MOCA Score & $23.6 \pm 11$ & $16.8 \pm 8.3$ & $<.001$ & N/A & N/A & \\
\hline FRS \% & N/A & N/A & & $88.01 \pm 28.7$ & $33.5 \pm 26.6$ & $<.001$ \\
\hline Letter Fluency Z-score & $0.7 \pm 0.7$ & $-0.9 \pm 0.6$ & $<.001$ & $-0.03 \pm 1$ & $-1.3 \pm 1.4$ & $<.001$ \\
\hline $\begin{array}{l}\text { Semantic Fluency Z- } \\
\text { score }\end{array}$ & $0.6 \pm 0.6$ & $-1.06 \pm 0.7$ & $<.001$ & $0.1 \pm 1$ & $-2.2 \pm 1.02$ & $<.001$ \\
\hline Genetic Group C9orf72 & - & - & & $87(35.2 \%)$ & $39(52 \%)$ & \\
\hline MAPT & - & - & & $40(16.2 \%)$ & $17(22.7 \%)$ & \\
\hline GRN & - & - & & $120(48.6 \%)$ & $19(25.3 \%)$ & \\
\hline
\end{tabular}

NOTE: Values expressed as mean \pm standard deviation, median [interquartile range]. Data available is specified for each clinical variable as N. N/A: data not available from the original databases.

Abbreviations: FTLD: frontotemporal lobar degeneration neuroimaging initiative; GENFI: genetic frontotemporal dementia initiative; bvFTD: behavioural-variant frontotemporal dementia. CNCs: cognitively normal controls;

MMSE: Mini Mental State Examination. FTLD-CDR: Frontotemporal lobar degeneration clinical dementia rating. MOCA: Montreal Cognitive Assessment. CGI: Clinical Global Impression. FRS: Frontotemporal dementia rating scale.

\subsection{Voxel-wise DBM group differences.}

Greater gray and white matter atrophy were found in the medial and inferior lateral portions of the frontal lobes as well as dorsolateral prefrontal cortex, insula, basal ganglia, medial and anterior temporal regions bilaterally and regions of brainstem and cerebellum in bvFTD. Correspondingly, volume increase was shown in the ventricles and sulci, being more evident in frontal horns and lateral sulcus (34).

\subsection{Random forest classification}

\subsubsection{Cross-validation results within the training cohort}


The accuracy achieved for discrimination between bvFTD and CNCs using solely morphometric MRI features (DBM) was $89 \%$, with a sensitivity of $82 \%$ and specificity of $93 \%$. When adding one cognitive score (i.e., DBM+SF) the classifier accuracy reached $94 \%$, with $89 \%$ sensitivity and $98 \%$ specificity.

\subsubsection{Classification within the validation cohort using solely $D B M$ and $D B M+S F$}

The application of the random forest classification model based on the training cohort to the validation cohort resulted in an accuracy of $88 \%$ when discriminating bvFTD patients from CNCs. Sensitivity and specificity were $81 \%$ and $92 \%$, respectively using a probability score with an optimal cut point of 0.4 as threshold. This led to a positive likelihood ratio (LR+) of 10.13 and negative likelihood ratio (LR-) of 0.21 .

The inclusion of semantic fluency in the classification model resulted in an accuracy of $91 \%$, sensitivity of $79 \%$ and specificity of $96 \%$; resulting in LR+ of 19.75 and LR- of 0.22 . The ROC for DBM and DBM + SF classifiers are shown in Figure 1. Figure 2 shows the true positive rates for bvFTD and CNCs according the probability score for DBM (panel A) and DBM+SF (panel B). Table 2 shows the corresponding accuracy, sensitivity, specificity and likelihood ratios for the two models (DBM and DBM+SF) using different thresholds on the probability scores (e.g., for probability scores $>0.4)$. 
bioRxiv preprint doi: https://doi.org/10.1101/2019.12.19.883462; this version posted December 20, 2019. The copyright holder for this preprint (which was not certified by peer review) is the author/funder, who has granted bioRxiv a license to display the preprint in perpetuity. It is made available under aCC-BY-ND 4.0 International license.

Figure 1. Receiver operating characteristic curves (ROC) for DBM and DBM+SF classifiers

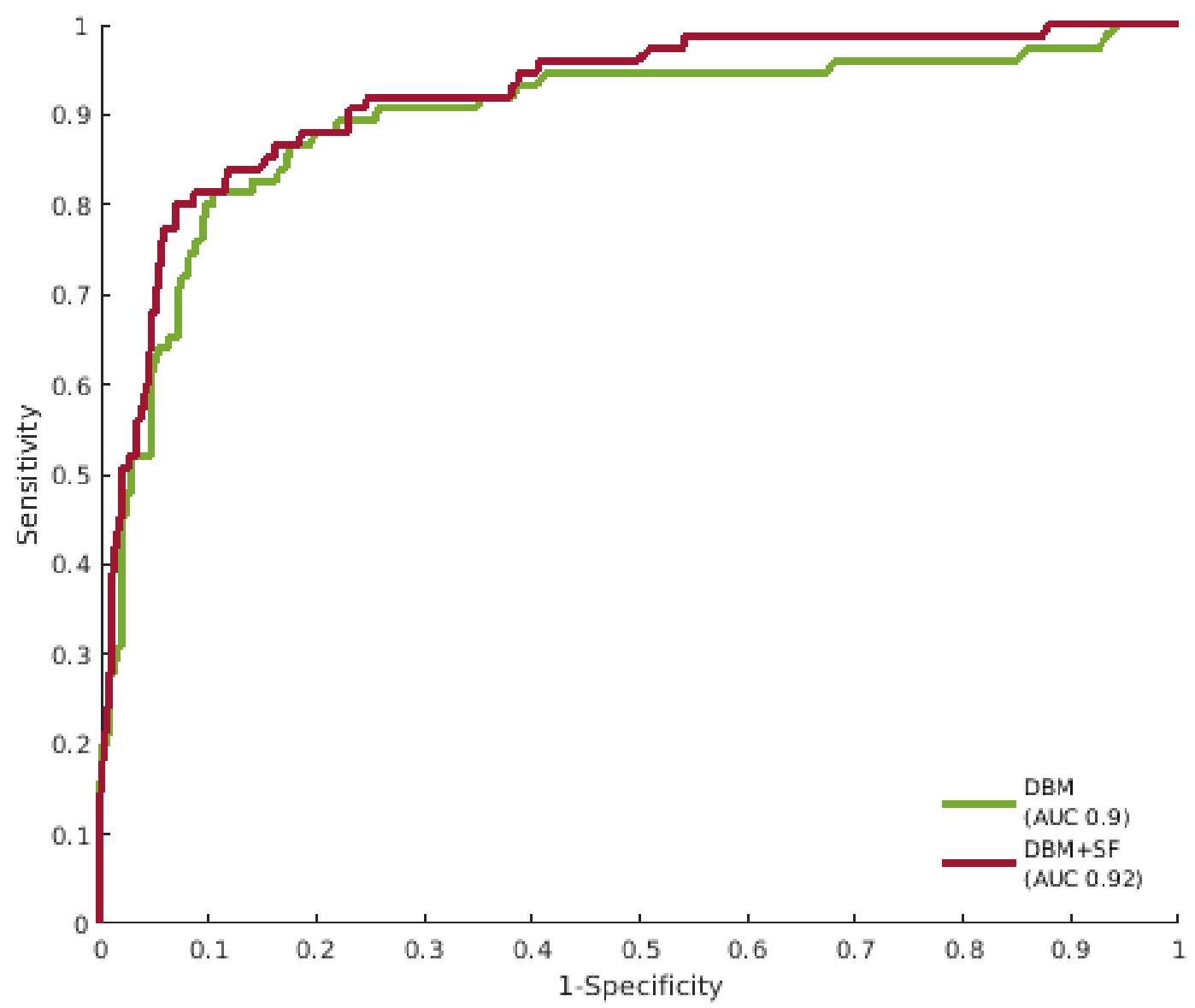

Abbreviations: DBM: deformation-based morphometry; SF: semantic fluency; AUC: area under the curve 
bioRxiv preprint doi: https://doi.org/10.1101/2019.12.19.883462; this version posted December 20, 2019. The copyright holder for this preprint (which was not certified by peer review) is the author/funder, who has granted bioRxiv a license to display the preprint in perpetuity. It is made available under aCC-BY-ND 4.0 International license.

Figure 2. True positive rates for bvFTD and controls according to the probability score threshold for classification using DBM (panel A) or DBM + SF (panel B)

A
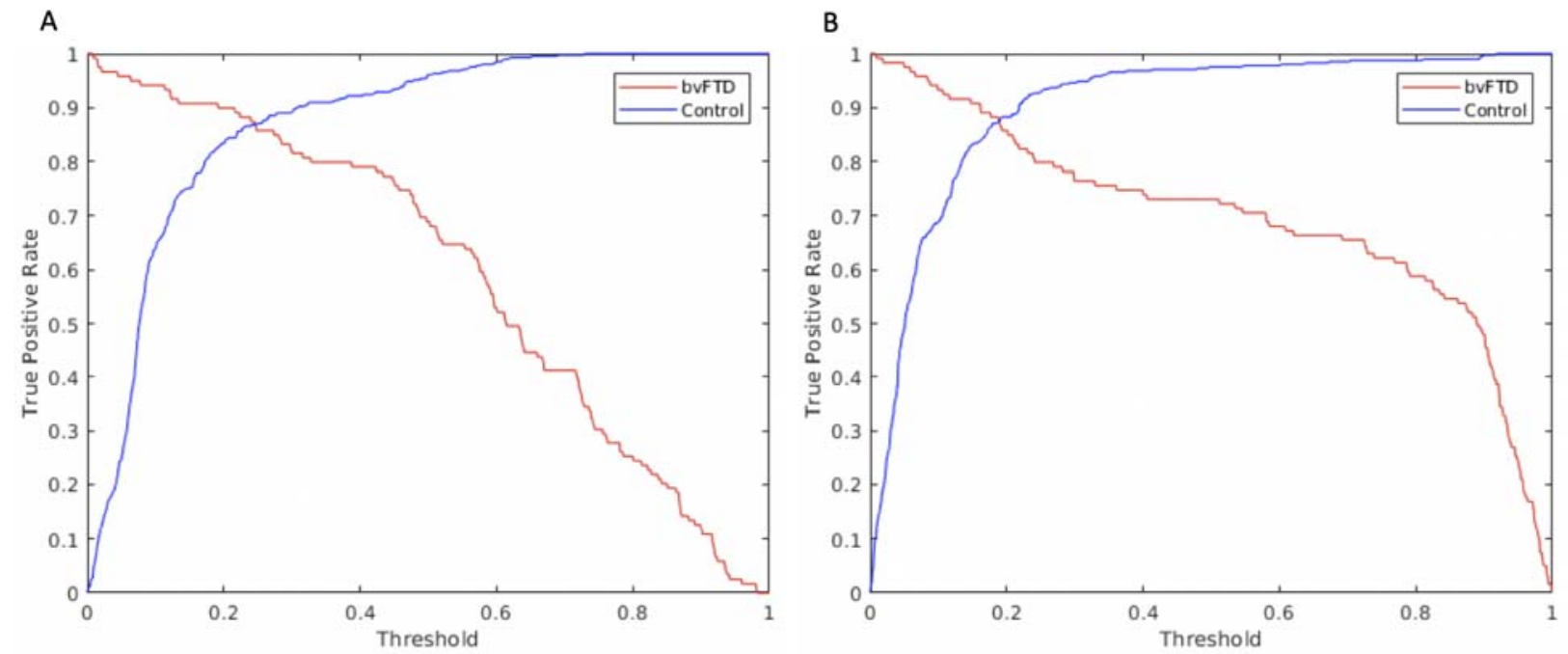

Abbreviations: bvFTD: behavioural-variant frontotemporal dementia. CNCs: cognitively normal controls 
Table 2. Classification performance using DBM and DBM + SF

\begin{tabular}{lllllll} 
& & Accuracy & Sensitivity & Specificity & LR+ & LR- \\
\hline $\begin{array}{l}\text { Probability score } \\
\text { Threshold* }\end{array}$ & & & & & & \\
& & & & & & \\
& $\mathbf{0 . 6 0}$ & 0.90 & 0.53 & 0.98 & 26.50 & 0.48 \\
& $\mathbf{0 . 5 5}$ & 0.91 & 0.64 & 0.97 & 21.33 & 0.37 \\
& $\mathbf{0 . 5 0}$ & 0.90 & 0.71 & 0.96 & 17.75 & 0.30 \\
& $\mathbf{0 . 4 5}$ & 0.89 & 0.77 & 0.93 & 11.00 & 0.25 \\
& $\mathbf{0 . 4 0}$ & 0.88 & 0.81 & 0.92 & 10.13 & 0.21 \\
& $\mathbf{0 . 3 5}$ & 0.86 & 0.81 & 0.91 & 9.00 & 0.21 \\
& $\mathbf{0 . 3 0}$ & 0.84 & 0.83 & 0.88 & 6.92 & 0.19 \\
Probability score & $\mathbf{0 . 2 5}$ & 0.81 & 0.87 & 0.87 & 6.69 & 0.15 \\
Threshold* & $\mathbf{0 . 2 0}$ & 0.78 & 0.89 & 0.83 & 5.24 & 0.13 \\
& $\mathbf{0 . 1 5}$ & 0.70 & 0.91 & 0.74 & 3.50 & 0.12 \\
& $\mathbf{0 . 1 0}$ & 0.60 & 0.95 & 0.64 & 2.64 & 0.08 \\
& & & & & & \\
& $\mathbf{0 . 6 0}$ & 0.92 & $\mathbf{D B M}+\mathbf{S F}$ & & & \\
& $\mathbf{0 . 5 5}$ & 0.92 & 0.71 & 0.98 & 35.50 & 0.30 \\
& $\mathbf{0 . 5 0}$ & 0.92 & 0.73 & 0.98 & 36.50 & 0.28 \\
& $\mathbf{0 . 4 5}$ & 0.91 & 0.77 & 0.98 & 38.50 & 0.23 \\
& $\mathbf{0 . 4 0}$ & $\mathbf{0 . 9 1}$ & 0.77 & 0.96 & 19.25 & 0.24 \\
& $\mathbf{0 . 3 5}$ & 0.91 & $\mathbf{0 . 7 9}$ & $\mathbf{0 . 9 6}$ & $\mathbf{1 9 . 7 5}$ & $\mathbf{0 . 2 2}$ \\
& $\mathbf{0 . 3 0}$ & 0.90 & 0.80 & 0.96 & 20.00 & 0.21 \\
& $\mathbf{0 . 2 5}$ & 0.88 & 0.80 & 0.95 & 16.00 & 0.21 \\
& $\mathbf{0 . 2 0}$ & 0.83 & 0.81 & 0.93 & 11.57 & 0.20 \\
& $\mathbf{0 . 1 5}$ & 0.78 & 0.87 & 0.87 & 6.69 & 0.15 \\
& $\mathbf{0 . 1 0}$ & 0.65 & 0.93 & 0.92 & 11.38 & 0.10 \\
& & & 0.68 & 2.91 & 0.10
\end{tabular}

NOTE: *Performances for each probability score threshold above which a subject is identified as bvFTD. Shaded rows correspond to the optimal cut-point estimated by Youden index.

Abbreviations: DBM: deformation-based morphometry; SF: semantic fluency score; LR+: positive likelihood ratio; LR-: negative likelihood ratio.

\subsubsection{False negative cases within the validation cohort}

The classification using DBM resulted in $19 \%$ of false negatives. These subjects were significantly younger than the bvFTD subjects correctly classified ( $57 \pm 10$ vs. $66 \pm 7$ years respectively, $P$ value $<.001)$ and the estimated time from onset was also smaller $(2 \pm 7$ years vs $6 \pm 5$ years; $P$ value $=.01)$. However, no significant differences were found in FTLD-CDR score between true positives and false negatives $(P$ value $=.07)$. The distribution of the genetic mutations did not show significant differences either between the false negatives and true 
positives. GRN corresponded to $22.7 \%$ of all false negatives and $25.4 \%$ of all true positives $(P$ value $=.7)$; for $C 9$ orf72 the distribution was $45.5 \%$ and $54.7 \%$ respectively $(P$ value $=.5)$ while for $M A P T$ it was $31.8 \%$ of the false negatives and $18.9 \%$ of the correctly classified bvFTD $(P$ value $=.3)$

\subsubsection{False positive cases within the validation cohort}

Only 10 out of $247 \mathrm{CNCs}$ (4\%) were erroneously classified as bvFTD. These subjects were significantly older than the subjects accurately classified as healthy subjects $(70 \pm 12$ years vs. $47 \pm 13$ years, respectively; $P$ value $<.001)$. No significant differences were found in the mean FTLD-CDR score $(P$ value $=.9)$. There was a small difference between the MMSE score for the false positives $(28.27 \pm 2.2)$ and for true negatives $(29.4 \pm 1 ; P$ value $<.001)$.

\subsubsection{Defining strategic cut-points}

Three cut-offs for both $\mathrm{DBM}$ and $\mathrm{DBM}+\mathrm{SF}$ were defined by giving consideration to the sensitivity, specificity, positive and negative likelihood ratios of different points of the ROC: 1) the optimal cut-point according to Youden index; 2) a sensitive (i.e., "rule-out") cut-point; and 3) a specific (i.e., "rule-in") cut-point (Figure 3). The sensitivity, specificity, LR- and LR+ expressed in the figure were estimated for each of these defined cut-points (e.g., for probability score $=0.4)$

Proposed thresholds for clinical decision-making for each classifier according to their likelihood ratios are proposed in Figure 3 (lower panels). A LR- $<0.1$ allows to reliably exclude (i.e., ruleout) bvFTD when the probability score is below 0.2 and 0.1 for DBM and DBM+SF, 
respectively. Probability scores over 0.4 for DBM and 0.25 for DBM+SF allow to confidently diagnose (i.e., rule-in) the disease with a $\mathrm{LR}+>10$. Corresponding likelihood ratios for different thresholds are shown in table 2.

Figure 3. Strategic ROC cut-points
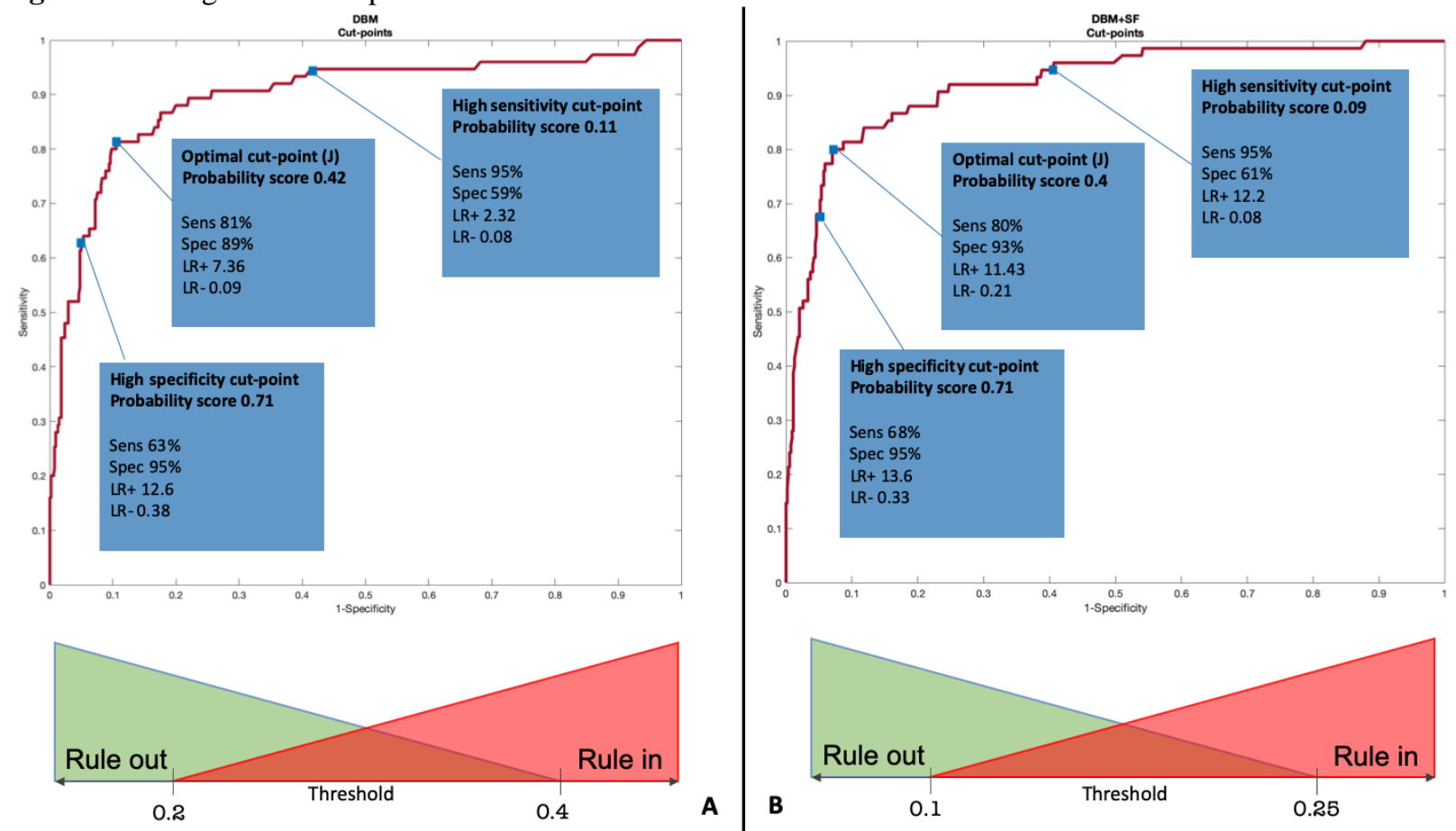

NOTE: A, upper panel: Cut-points for DBM classifier. A, lower panel: Thresholds for diagnosis using DBM classifier in relation to LR+ and LR-. B, upper panel: Cut-points for DBM+SF classifier. B, lower panel: Thresholds for diagnosis using DBM+SF classifier in relation to LR+ and LR-.

Abbreviations: DBM: deformation-based morphometry; SF: semantic fluency; J: Youden index; Sens: sensitivity; Spec: specificity; LR+: positive likelihood ratio; LR-: negative likelihood ratio

\section{Discussion}

In the present study we built a random forest classifier using morphometric MRI features for the individual prediction of bvFTD. The main findings are: 1) our random forest algorithm yielded areas under the curve of 0.90 and 0.92 using $\mathrm{DBM}$ and $\mathrm{DBM}+\mathrm{SF}$, respectively, in the independent validation cohort of genetically confirmed bvFTD cases; 2) the inclusion of a simple cognitive score (SF) improved the accuracies and specificity regardless of the probability 
threshold chosen, while reducing the false negative rate for probability scores $>0.5$; 3) we provide three cut-off values (a "statistically optimal" cut-point, a sensitive ("rule-out") cut-point and a specific ("rule-in") cut-point) for both DBM and DBM+SF classifiers; and 4) our results show good positive and negative likelihood ratios proving its reliability in ruling in and out the disease.

The likelihood ratio is the percentage of patients with a given test result divided by the percentage of controls with the same results. Meaning that ill people should be more likely to have an abnormal result of a given test than healthy individuals(35, 36). For DBM only classifier in the independent validation cohort, the optimal threshold yielded an area under the curve (AUC) of 0.9 with $81 \%$ sensitivity and $92 \%$ specificity leading to a positive LR+ of 10.13 and negative LR- of 0.21 . Whereas, the AUC, sensitivity and specificity using the DBM+SF model were $0.92,79 \%$ and $96 \%$, respectively. These values result in LR+ of 19.75 and LR- of 0.22 . To keep in mind, a test is moderately good at ruling in disease when $\mathrm{LR}+$ is greater than 2 and very good at doing it when LR+ is greater than 10 (37). Furthermore, a test is moderately good at ruling out the disease with LR- below 0.5 and very good below 0.1 . Hence, using the optimal thresholds, both models are very good at excluding non bvFTD subjects and moderately good at confirming the disease.

Our results show that the random forest classifier we developed in our training cohort can accurately predict bvFTD in individual subjects in a completely independent validation cohort coming from a different and independent database. Furthermore, the GENFI2 validation cohort includes bvFTD patients with a definite diagnosis (positive genetic mutation). Of note, our 
algorithm was able to accurately classify patients with genetic bvFTD even though they tend to have more atypical atrophy patterns(11). The performance of our classifier is superior than the performance reported in several articles that have analyzed the standard diagnostic methods currently used in the clinical practice. Within a pathology-confirmed cohort the sensitivity reported for the revised diagnostic criteria for bvFTD was $86 \%$ for possible diagnosis and $75 \%$ for probable bvFTD (with neuroimaging support) (7). However, these criteria reported a sensitivity of $85 \%$ and specificity of $27 \%$ for possible bvFTD diagnosis in a clinically relevant cohort of patients with mixed behavioral changes, reaching $82 \%$ specificity when adding a compatible MRI scan (38). Within a cohort with late onset behavioral disorders, $70 \%$ sensitivity and 93\% specificity have been reported for structural MRI alone for bvFTD assessed by an experienced neuroradiologist (8). The latter results have comparable positive and negative likelihood ratios to ours, even though our method does not rely on the expertise of the radiological observer.

Previous studies classified bvFTD from a control group (12-16). The best AUC was reported by Raamana et al (AUC 0.938, 100\% sensitivity and 88\% specificity). However, the main limitation of that study is that the bvFTD diagnosis from the validation cohort was based on clinical criteria (39). Contrarily, the bvFTD subjects from our validation cohort are known carriers of a pathogenic mutation and have therefore, definite bvFTD diagnosis.

The performance of the classifier was tested on a held-out database which included multi-center and multi-scanner data from different scanner models of both 1.5T and 3T field strengths. This further reinforces the generalizability (i.e., external validity) of our results and ensures their 
applicability in a clinical scenario with different scanners, even with different magnetic field strengths. This certainly constitutes one of the main strengths of this study along with the fact that our performance was estimated using one of the gold standards for FTD diagnosis (i.e., definite FTD supported by the presence of a pathogenic mutation). Of significant value, our algorithm is based on standard structural T1w MRI and a simple cognitive test that are already routinely acquired clinically, making for strong translational potential. On the other hand, the main limitation is that these results are yet to be validated prospectively in a clinically representative cohort including patients with diverse primary psychiatric disorders (a common differential diagnosis from bvFTD) (40). The classification accuracy also remains to be demonstrated in cohorts with other types of dementias and cardiovascular comorbidities, as these were uncommon in our dataset and could have influenced our very high specificity. Finally, in our results the false negatives/positives were significantly younger/older than the subjects that were correctly classified. This is likely due to the fact that the age range for the validation dataset (GENFI: minimum age: 39, maximum age:79) was larger than the training set (FTLDNI, minimum age 46, maximum age 75). Subjects that were outside the operating range of the classifier were therefore more likely to be misclassified. Adding subjects with similar ages to the training dataset will likely improve the results. In addition, specifically for the false negative cases, although the difference did not reach statistical significance $(\mathrm{p}=0.07)$, the false negatives had lower FTLD-CDR scores than the true positive cases, implying an earlier stage of the disease. It is plausible that such subjects with milder symptoms were not as well represented in NIFD given the difficulty of diagnosing bvFTD in the very mild stages when there is no known genetic mutation. 
To conclude, we propose an automatic method using structural MRI alone (already available and routinely used in the clinic) and including a simple cognitive test that could be administered by any physician in few minutes for reliable individual prediction of bvFTD at the individual subject level. If validated in a prospective study, this algorithm has the potential to improve diagnostic accuracy, particularly in setting without access to specialized FTD care. 


\section{Acknowledgements}

We would like to acknowledge funding from the Famille Louise \& André Charron.

Data collection and sharing for this project was funded by the Frontotemporal Lobar Degeneration Neuroimaging Initiative (National Institutes of Health Grant R01 AG032306). The study is coordinated through the University of California, San Francisco, Memory and Aging Center. FTLDNI data are disseminated by the Laboratory for Neuro Imaging at the University of Southern California.

Brain scan acquisition at the McConnell Brain Imaging was supported by the Brain Canada Foundation with support from Health Canada and the Canada Foundation for Innovation (CFI Project 34874).

This work was supported by Italian Ministry of Health (CoEN015 and Ricerca Corrente).

\section{Declaration of interests}

Dr. Manera reports no disclosures

Dr. Dadar reports no disclosures

Dr. Collins is co-founder of True Positive Medical Devices.

Dr. Ducharme receives salary funding from the Fonds de Recherche du Québec - Santé. Dr. Ducharme is the co-founder of Arctic Fox AI.

Dr. Ghidoni reports no disclosures 


\section{Appendix:}

\section{List of other GENFI consortium members:}

Sónia Afonso - Instituto Ciencias Nucleares Aplicadas a Saude, Universidade de Coimbra, Coimbra, Portugal Maria Rosario Almeida - Centre of Neurosciences and Cell Biology, Universidade de Coimbra, Coimbra, Portugal Sarah Anderl-Straub - Department of Neurology, Ulm University, Ulm, Germany

Christin Andersson - Department of Clinical Neuroscience, Karolinska Institutet, Stockholm, Sweden

Anna Antonell - Alzheimer's disease and other cognitive disorders unit, Neurology Department, Hospital Clinic, Institut d'Investigacions Biomèdiques, Barcelona, Spain

Silvana Archetti - Biotechnology Laboratory, Department of Diagnostics, ASST Brescia Hospital, Brescia, Italy

Andrea Arighi - Fondazione IRCSS Ca’ Granda, Ospedale Maggiore Policlinico, Neurodegenerative Diseases Unit, Milan, Italy

Mircea Balasa - Alzheimer's disease and other cognitive disorders unit, Neurology Department, Hospital Clinic, Institut d'Investigacions Biomèdiques, Barcelona, Spain

Myriam Barandiaran - Neuroscience Area, Biodonostia Health Research Institute, Paseo Dr Begiristain sn, CP 20014, San Sebastian, Gipuzkoa, Spain

Nuria Bargalló - Radiology Department, Image Diagnosis Center, Hospital Clínic and Magnetic Resonance Image core facility, IDIBAPS, Barcelona, Spain

Robart Bartha - Department of Medical Biophysics, Robarts Research Institute, University of Western Ontario, London, Ontario, Canada

Benjamin Bender - Department of Diagnostic and Interventional Neuroradiology, University of Tuebingen, Tuebingen, Germany

Alberto Benussi - Centre for Neurodegenerative Disorders, Department of Clinical and Experimental Sciences, University of Brescia, Italy

Luisa Benussi - Istituto di Ricovero e Cura a Carattere Scientifico Istituto Centro San Giovanni di Dio Fatebenefratelli, Brescia, Italy

Valentina Bessi - Department of Neuroscience, Psychology, Drug Research, and Child Health, University of

Florence, Florence, Italy

Giuliano Binetti - Istituto di Ricovero e Cura a Carattere Scientifico Istituto Centro San Giovanni di Dio Fatebenefratelli, Brescia, Italy

Sandra Black - LC Campbell Cognitive Neurology Research Unit, Sunnybrook Research Institute, Toronto, Canada Martina Bocchetta - Dementia Research Centre, Department of Neurodegenerative Disease, UCL Institute of Neurology, Queen Square London, UK

Sergi Borrego-Ecija - Alzheimer's disease and other cognitive disorders unit, Neurology Department, Hospital Clinic, Institut d'Investigacions Biomèdiques, Barcelona, Spain

Jose Bras - Dementia Research Institute, Department of Neurodegenerative Disease, UCL Institute of Neurology, Queen Square, London, UK

Rose Bruffaerts - Laboratory for Cognitive Neurology, Department of Neurosciences, KU Leuven, Leuven, Belgium Paola Caroppo - Fondazione Istituto di Ricovero e Cura a Carattere Scientifico Istituto Neurologico Carlo Besta, Milan, Italy

David Cash - Dementia Research Centre, Department of Neurodegenerative Disease, UCL Institute of Neurology, Queen Square, London, UK

Miguel Castelo-Branco - Neurology Department, Centro Hospitalar e Universitário de Coimbra, Instituto de Ciências Nucleares Aplicadas à Saúde (ICNAS), Coimbra, Portugal

Rhian Convery-Dementia Research Centre, Department of Neurodegenerative Disease, UCL Institute of Neurology, Queen Square, London, UK

Thomas Cope - Department of Clinical Neuroscience, University of Cambridge, Cambridge, UK Maura Cosseddu - Neurology, ASST Brescia Hospital, Brescia, Italy 
María de Arriba - Neuroscience Area, Biodonostia Health Research Institute, Paseo Dr Begiristain sn, CP 20014, San Sebastian, Gipuzkoa, Spain

Giuseppe Di Fede - Fondazione Istituto di Ricovero e Cura a Carattere Scientifico Istituto Neurologico Carlo Besta, Milan, Italy

Zigor Díaz - CITA Alzheimer, San Sebastian, Spain

Diana Duro - Faculty of Medicine, Universidade de Coimbra, Coimbra, Portugal

Chiara Fenoglio - University of Milan, Centro Dino Ferrari, Milan, Italy

Camilla Ferrari - Department of Neuroscience, Psychology, Drug Research, and Child Health, University of

Florence, Florence, Italy

Carlos Ferreira - Instituto Ciências Nucleares Aplicadas à Saúde, Universidade de Coimbra, Coimbra, Portugal

Catarina B. Ferreira - Faculty of Medicine, University of Lisbon, Lisbon, Portugal

Toby Flanagan - Faculty of Biology, Medicine and Health, Division of Neuroscience and Experimental Psychology, University of Manchester, Manchester, UK

Nick Fox - Dementia Research Centre, Department of Neurodegenerative Disease, UCL Institute of Neurology, Queen Square, London, UK

Morris Freedman - Division of Neurology, Baycrest Centre for Geriatric Care, University of Toronto, Toronto, Canada

Giorgio Fumagalli - Fondazione IRCSS Ca' Granda, Ospedale Maggiore Policlinico, Neurodegenerative Diseases Unit, Milan, Italy; Department of Neuroscience, Psychology, Drug Research and Child Health, University of Florence, Florence, Italy

Alazne Gabilondo - Neuroscience Area, Biodonostia Health Research Institute, Paseo Dr Begiristain sn, CP 20014, San Sebastian, Gipuzkoa, Spain

Roberto Gasparotti - Neuroradiology Unit, University of Brescia, Brescia, Italy

Serge Gauthier - Department of Neurology and Neurosurgery, McGill University, Montreal, Québec, Canada

Stefano Gazzina - Neurology, ASST Brescia Hospital, Brescia, Italy

Giorgio Giaccone - Fondazione Istituto di Ricovero e Cura a Carattere Scientifico Istituto Neurologico Carlo Besta, Milan, Italy

Ana Gorostidi - Neuroscience Area, Biodonostia Health Research Institute, Paseo Dr Begiristain sn, CP 20014, San Sebastian, Gipuzkoa, Spain

Caroline Greaves - Dementia Research Centre, Department of Neurodegenerative Disease, UCL Institute of Neurology, Queen Square London, UK

Rita Guerreiro - Dementia Research Institute, Department of Neurodegenerative Disease, UCL Institute of Neurology, London, UK

Carolin Heller - Dementia Research Centre, Department of Neurodegenerative Disease, UCL Institute of Neurology, Queen Square, London, UK

Tobias Hoegen - Department of Neurology, Ludwig-Maximilians-University of Munich, Munich, Germany

Begoña Indakoetxea - Cognitive Disorders Unit, Department of Neurology, Donostia University Hospital, Paseo Dr

Begiristain sn, CP 20014, San Sebastian, Gipuzkoa, Spain

Vesna Jelic - Division of Clinical Geriatrics, Karolinska Institutet, Stockholm, Sweden

Lize Jiskoot - Department of Neurology, Erasmus Medical Center, Rotterdam, The Netherlands

Hans-Otto Karnath - Section of Neuropsychology, Department of Cognitive Neurology, Center for Neurology \& Hertie-Institute for Clinical Brain Research, Tübingen, Germany

Ron Keren - University Health Network Memory Clinic, Toronto Western Hospital, Toronto, Canada

Maria João Leitão - Centre of Neurosciences and Cell Biology, Universidade de Coimbra, Coimbra, Portugal

Albert Lladó - Alzheimer's disease and other cognitive disorders unit, Neurology Department, Hospital Clinic, Institut d'Investigacions Biomèdiques, Barcelona, Spain

Gemma Lombardi - Department of Neuroscience, Psychology, Drug Research and Child Health, University of Florence, Florence, Italy

Sandra Loosli - Department of Neurology, Ludwig-Maximilians-University of Munich, Munich, Germany 
Carolina Maruta - Lisbon Faculty of Medicine, Language Research Laboratory, Lisbon, Portugal

Simon Mead - MRC Prion Unit, Department of Neurodegenerative Disease, UCL Institute of Neurology, Queen Square, London, UK

Lieke Meeter - Department of Neurology, Erasmus Medical Center, Rotterdam, Netherlands

Gabriel Miltenberger - Faculty of Medicine, University of Lisbon, Lisbon, Portugal

Rick van Minkelen - Department of Clinical Genetics, Erasmus Medical Center, Rotterdam, The Netherlands

Sara Mitchell - LC Campbell Cognitive Neurology Research Unit, Sunnybrook Research Institute, Toronto, Canada

Katrina M Moore - Dementia Research Centre, Department of Neurodegenerative Disease, UCL Institute of Neurology, Queen Square, London, UK

Benedetta Nacmias - Department of Neuroscience, Psychology, Drug Research and Child Health, University of Florence, Florence, Italy

Mollie Neason - Dementia Research Centre, Department of Neurodegenerative Disease, UCL Institute of Neurology, Queen Square, London, UK

Jennifer Nicholas - Department of Medical Statistics, London School of Hygiene and Tropical Medicine, London, UK

Linn Öijerstedt - Department of Geriatric Medicine, Karolinska Institutet, Stockholm, Sweden

Jaume Olives - Alzheimer's disease and other cognitive disorders unit, Neurology Department, Hospital Clinic, Institut d'Investigacions Biomèdiques, Barcelona, Spain

Sebastien Ourselin - School of Biomedical Engineering \& Imaging Sciences, King's College London, London, UK

Alessandro Padovani - Centre for Neurodegenerative Disorders, Department of Clinical and Experimental Sciences, University of Brescia, Italy

Jessica Panman - Department of Neurology, Erasmus Medical Center, Rotterdam, The Netherlands

Janne Papma - Department of Neurology, Erasmus Medical Center, Rotterdam, The Netherlands

Georgia Peakman - Department of Neurodegenerative Disease, Dementia Research Centre, UCL Institute of Neurology, Queen Square, London, UK

Irene Piaceri - Department of Neuroscience, Psychology, Drug Research and Child Health, University of Florence, Florence

Michela Pievani - Istituto di Ricovero e Cura a Carattere Scientifico Istituto Centro San Giovanni di Dio Fatebenefratelli, Brescia, Italy

Yolande Pijnenburg - VUMC, Amsterdam, The Netherlands

Cristina Polito - Department of Biomedical, Experimental and Clinical Sciences "Mario Serio", Nuclear Medicine Unit, University of Florence, Florence, Italy

Enrico Premi - Stroke Unit, ASST Brescia Hospital, Brescia, Italy

Sara Prioni - Fondazione Istituto di Ricovero e Cura a Carattere Scientifico Istituto Neurologico Carlo Besta, Milan, Italy

Catharina Prix - Department of Neurology, Ludwig-Maximilians-University Munich, Germany

Rosa Rademakers - Department of Neurosciences, Mayo Clinic, Jacksonville, Florida, USA

Veronica Redaelli - Fondazione Istituto di Ricovero e Cura a Carattere Scientifico Istituto Neurologico Carlo Besta, Milan, Italy

Tim Rittman - Department of Clinical Neurosciences, University of Cambridge, Cambridge, UK

Ekaterina Rogaeva - Tanz Centre for Research in Neurodegenerative Diseases, University of Toronto, Toronto, Canada

Pedro Rosa-Neto - Translational Neuroimaging Laboratory, McGill University Montreal, Québec, Canada

Giacomina Rossi - Fondazione Istituto di Ricovero e Cura a Carattere Scientifico Istituto Neurologico Carlo Besta, Milan, Italy

Martin Rossor - Dementia Research Centre, Department of Neurodegenerative Disease, UCL Institute of Neurology, Queen Square, London, UK

Beatriz Santiago - Neurology Department, Centro Hospitalar e Universitário de Coimbra, Coimbra, Portugal 
Elio Scarpini - University of Milan, Centro Dino Ferrari, Milan, Italy; Fondazione IRCSS Ca' Granda, Ospedale Maggiore Policlinico, Neurodegenerative Diseases Unit, Milan, Italy

Sonja Schönecker - Neurologische Klinik, Ludwig-Maximilians-Universität München, Munich, Germany

Elisa Semler - Department of Neurology, Ulm University, Ulm, Germany

Rachelle Shafei - Dementia Research Centre, Department of Neurodegenerative Disease, UCL Institute of Neurology, Queen Square, London, UK

Christen Shoesmith - Department of Clinical Neurological Sciences, University of Western Ontario, London, Ontario, Canada

Miguel Tábuas-Pereira - Centre of Neurosciences and Cell Biology, Universidade de Coimbra, Coimbra, Portugal

Mikel Tainta - Neuroscience Area, Biodonostia Health Research Institute, Paseo Dr Begiristain sn, CP 20014, San Sebastian, Gipuzkoa, Spain

Ricardo Taipa - Neuropathology Unit and Department of Neurology, Centro Hospitalar do Porto - Hospital de Santo António, Oporto, Portugal

David Tang-Wai - University Health Network Memory Clinic, Toronto Western Hospital, Toronto, Canada

David L Thomas - Neuroradiological Academic Unit, UCL Institute of Neurology, London, UK

Hakan Thonberg - Center for Alzheimer Research, Division of Neurogeriatrics, Karolinska Institutet, Stockholm, Sweden

Carolyn Timberlake - Department of Clinical Neurosciences, University of Cambridge, Cambridge, UK

Pietro Tiraboschi - Fondazione Istituto di Ricovero e Cura a Carattere Scientifico Istituto Neurologico Carlo Besta, Milano, Italy

Emily Todd - Department of Neurodegenerative Disease, Dementia Research Centre, UCL Institute of Neurology, Queen Square, London, UK

Philip Vandamme - Neurology Service, University Hospitals Leuven, Belgium; Laboratory for Neurobiology, VIBKU Leuven Centre for Brain Research, Leuven, Belgium

Mathieu Vandenbulcke - Geriatric Psychiatry Service, University Hospitals Leuven, Belgium; Neuropsychiatry, Department of Neurosciences, KU Leuven, Leuven, Belgium

Michele Veldsman - Nuffield Department of Clinical Neurosciences, Medical Sciences Division, University of Oxford, UK

Ana Verdelho - Department of Neurosciences, Santa Maria Hospital, University of Lisbon, Portugal

Jorge Villanua - OSATEK Unidad de Donostia, San Sebastian, Gipuzkoa, Spain

Jason Warren - Dementia Research Centre, Department of Neurodegenerative Disease, UCL Institute of Neurology, Queen Square, London, UK

Carlo Wilke - Hertie Institute for Clinical Brain Research, University of Tuebingen, Tuebingen, Germany

Ione Woollacott - Dementia Research Centre, Department of Neurodegenerative Disease, UCL Institute of

Neurology, Queen Square, London, UK

Elisabeth Wlasich - Neurologische Klinik, Ludwig-Maximilians-Universität München, Munich, Germany

Henrik Zetterberg - Department of Neurodegenerative Disease, UCL Institute of Neurology, London, UK

Miren Zulaica - Neuroscience Area, Biodonostia Health Research Institute, Paseo Dr Begiristain sn, CP 20014, San

Sebastian, Gipuzkoa, Spain. 


\section{References:}

1. Seelaar H, Rohrer JD, Pijnenburg YA, Fox NC, van Swieten JC. Clinical, genetic and pathological heterogeneity of frontotemporal dementia: a review. J Neurol Neurosurg Psychiatry. 2011;82(5):476-86.

2. Onyike CU, Diehl-Schmid J. The epidemiology of frontotemporal dementia. Int Rev Psychiatry. 2013;25(2):130-7.

3. Rohrer JD, Guerreiro R, Vandrovcova J, Uphill J, Reiman D, Beck J, et al. The heritability and genetics of frontotemporal lobar degeneration. Neurology. 2009;73(18):1451-6.

4. Cairns NJ, Bigio EH, Mackenzie IR, Neumann M, Lee VM, Hatanpaa KJ, et al. Neuropathologic diagnostic and nosologic criteria for frontotemporal lobar degeneration: consensus of the Consortium for Frontotemporal Lobar Degeneration. Acta Neuropathol. 2007;114(1):5-22.

5. Bang J, Spina S, Miller BL. Frontotemporal dementia. Lancet. 2015;386(10004):167282.

6. Espay AJ, Litvan I. Parkinsonism and frontotemporal dementia: the clinical overlap. J Mol Neurosci. 2011;45(3):343-9.

7. Rascovsky K, Hodges JR, Knopman D, Mendez MF, Kramer JH, Neuhaus J, et al. Sensitivity of revised diagnostic criteria for the behavioural variant of frontotemporal dementia. Brain. 2011;134(Pt 9):2456-77.

8. Vijverberg EG, Wattjes MP, Dols A, Krudop WA, Moller C, Peters A, et al. Diagnostic Accuracy of MRI and Additional [18F]FDG-PET for Behavioral Variant Frontotemporal Dementia in Patients with Late Onset Behavioral Changes. J Alzheimers Dis. 2016;53(4):128797.

9. Whitwell JL, Boeve BF, Weigand SD, Senjem ML, Gunter JL, Baker MC, et al. Brain atrophy over time in genetic and sporadic frontotemporal dementia: a study of 198 serial magnetic resonance images. Eur J Neurol. 2015;22(5):745-52.

10. Whitwell JL, Weigand SD, Boeve BF, Senjem ML, Gunter JL, DeJesus-Hernandez M, et al. Neuroimaging signatures of frontotemporal dementia genetics: C9ORF72, tau, progranulin and sporadics. Brain. 2012;135(Pt 3):794-806.

11. Cash DM, Bocchetta M, Thomas DL, Dick KM, van Swieten JC, Borroni B, et al. Patterns of gray matter atrophy in genetic frontotemporal dementia: results from the GENFI study. Neurobiol Aging. 2018;62:191-6.

12. Chow TW, Binns MA, Freedman M, Stuss DT, Ramirez J, Scott CJ, et al. Overlap in frontotemporal atrophy between normal aging and patients with frontotemporal dementias. Alzheimer Dis Assoc Disord. 2008;22(4):327-35.

13. Mahoney CJ, Ridgway GR, Malone IB, Downey LE, Beck J, Kinnunen KM, et al. Profiles of white matter tract pathology in frontotemporal dementia. Hum Brain Mapp. 2014;35(8):4163-79.

14. Meyer S, Mueller K, Stuke K, Bisenius S, Diehl-Schmid J, Jessen F, et al. Predicting behavioral variant frontotemporal dementia with pattern classification in multi-center structural MRI data. Neuroimage Clin. 2017;14:656-62.

15. Moller C, Pijnenburg YA, van der Flier WM, Versteeg A, Tijms B, de Munck JC, et al. Alzheimer Disease and Behavioral Variant Frontotemporal Dementia: Automatic Classification Based on Cortical Atrophy for Single-Subject Diagnosis. Radiology. 2016;279(3):838-48. 
16. Raamana PR, Rosen H, Miller B, Weiner MW, Wang L, Beg MF. Three-Class Differential Diagnosis among Alzheimer Disease, Frontotemporal Dementia, and Controls. Front Neurol. 2014;5:71.

17. Canu E, Agosta F, Mandic-Stojmenovic G, Stojkovic T, Stefanova E, Inuggi A, et al. Multiparametric MRI to distinguish early onset Alzheimer's disease and behavioural variant of frontotemporal dementia. Neuroimage Clin. 2017;15:428-38.

18. Frings L, Yew B, Flanagan E, Lam BY, Hull M, Huppertz HJ, et al. Longitudinal grey and white matter changes in frontotemporal dementia and Alzheimer's disease. PLoS One. 2014;9(3):e90814.

19. Wang J, Redmond SJ, Bertoux M, Hodges JR, Hornberger M. A Comparison of Magnetic Resonance Imaging and Neuropsychological Examination in the Diagnostic Distinction of Alzheimer's Disease and Behavioral Variant Frontotemporal Dementia. Front Aging Neurosci. 2016;8:119.

20. Zhutovsky P, Vijverberg EGB, Bruin WB, Thomas RM, Wattjes MP, Pijnenburg YAL, et al. Individual Prediction of Behavioral Variant Frontotemporal Dementia Development Using Multivariate Pattern Analysis of Magnetic Resonance Imaging Data. J Alzheimers Dis. 2019;68(3):1229-41.

21. Breiman L. Random Forests. Machine Learning. 2001;45(1):5-32.

22. Rohrer JD, Nicholas JM, Cash DM, van Swieten J, Dopper E, Jiskoot L, et al. Presymptomatic cognitive and neuroanatomical changes in genetic frontotemporal dementia in the Genetic Frontotemporal dementia Initiative (GENFI) study: a cross-sectional analysis. Lancet Neurol. 2015;14(3):253-62.

23. Aubert-Broche B, Fonov VS, Garcia-Lorenzo D, Mouiha A, Guizard N, Coupe P, et al. A new method for structural volume analysis of longitudinal brain MRI data and its application in studying the growth trajectories of anatomical brain structures in childhood. Neuroimage. 2013;82:393-402.

24. Coupe P, Yger P, Prima S, Hellier P, Kervrann C, Barillot C. An optimized blockwise nonlocal means denoising filter for 3-D magnetic resonance images. IEEE Trans Med Imaging. 2008;27(4):425-41.

25. Sled JG, Zijdenbos AP, Evans AC. A nonparametric method for automatic correction of intensity nonuniformity in MRI data. IEEE Trans Med Imaging. 1998;17(1):87-97.

26. Collins DL, Neelin P, Peters TM, Evans AC. Automatic 3D intersubject registration of MR volumetric data in standardized Talairach space. J Comput Assist Tomogr. 1994;18(2):192205.

27. A.C.Evans DLCa. Animal: validation and applications of nonlinear registration-based segmentation. International Journal ofPattern Recognition and Artificial Intelligence. 1997;11(08):1271-94.

28. Avants BB, Epstein CL, Grossman M, Gee JC. Symmetric diffeomorphic image registration with cross-correlation: evaluating automated labeling of elderly and neurodegenerative brain. Med Image Anal. 2008;12(1):26-41.

29. Ashburner J, Friston KJ. Voxel-based morphometry--the methods. Neuroimage. 2000;11(6 Pt 1):805-21.

30. Ashburner J, Hutton C, Frackowiak R, Johnsrude I, Price C, Friston K. Identifying global anatomical differences: deformation-based morphometry. Hum Brain Mapp. 1998;6(5-6):348-57. 
31. Libon DJ, McMillan C, Gunawardena D, Powers C, Massimo L, Khan A, et al. Neurocognitive contributions to verbal fluency deficits in frontotemporal lobar degeneration. Neurology. 2009;73(7):535-42.

32. Giovagnoli AR, Erbetta A, Reati F, Bugiani O. Differential neuropsychological patterns of frontal variant frontotemporal dementia and Alzheimer's disease in a study of diagnostic concordance. Neuropsychologia. 2008;46(5):1495-504.

33. Hornberger M, Piguet O, Kipps C, Hodges JR. Executive function in progressive and nonprogressive behavioral variant frontotemporal dementia. Neurology. 2008;71(19):1481-8.

34. Manera AL, Dadar M, Collins DL, Ducharme S. Deformation based morphometry study of longitudinal MRI changes in behavioral variant frontotemporal dementia. Neuroimage: Clinical. 2019;24.

35. Grimes DA, Schulz KF. Refining clinical diagnosis with likelihood ratios. Lancet. 2005;365(9469):1500-5.

36. McGee S. Simplifying likelihood ratios. J Gen Intern Med. 2002;17(8):646-9.

37. Doust J. Using probabilistic reasoning. BMJ. 2009;339.

38. Vijverberg EG, Dols A, Krudop WA, Peters A, Kerssens CJ, van Berckel BN, et al. Diagnostic Accuracy of the Frontotemporal Dementia Consensus Criteria in the Late-Onset Frontal Lobe Syndrome. Dement Geriatr Cogn Disord. 2016;41(3-4):210-9.

39. McCarthy J, Collins DL, Ducharme S. Morphometric MRI as a diagnostic biomarker of frontotemporal dementia: A systematic review to determine clinical applicability. Neuroimage Clin. 2018;20:685-96.

40. Ducharme S, Price BH, Larvie M, Dougherty DD, Dickerson BC. Clinical Approach to the Differential Diagnosis Between Behavioral Variant Frontotemporal Dementia and Primary Psychiatric Disorders. Am J Psychiatry. 2015;172(9):827-37. 